\title{
The Role of Serotonin in Low Level Visual Processes, as Revealed by Using an "Ecstasy" (MDMA)-Based Research Model
}

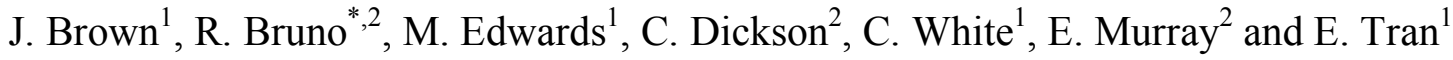 \\ ${ }^{I}$ School of Psychology, Australian National University, Australia \\ ${ }^{2}$ School of Psychology, University of Tasmania, Australia
}

Keywords: Ecstasy, MDMA, amphetamine, vision, primary visual cortex, motion, serotonin.

\section{INTRODUCTION}

A growing body of evidence suggests that regular 'ecstasy' (3, 4-methylenedioxymethamphetamine; MDMA) use causes lasting changes to central serotonergic functioning in humans, including in the occipital lobe. Brown, Edwards, et al. [1] proposed that MDMA-related serotonergic changes in the occipital lobe may reduce lateral inhibition between occipital neurons involved in low level visual perception, resulting in broadening the tuning bandwidths of those neurons.

\section{METHODS, RESULTS AND DISCUSSION}

This was tested using the tilt-aftereffect illusion, whereby adaptation of V1 orientation-sensitive neurons to a visual stimulus consisting of parallel lines angled at, for example, $15^{\circ}$ to the right of vertical results in subsequently presented vertical lines appearing to be orientated slightly to the left of vertical. Brown et al. found that this effect was larger in ecstasy users than non-drug using controls, but only in a subset that had not recently used amphetamines. This effect was apparent following an adaptation angle of $40^{\circ}$ but not $15^{\circ}$, and was interpreted in terms of lateral inhibition. This finding has been subsequently replicated and extended by Dickson, Bruno and Brown [2], who found the effect at $15^{\circ}$, $30^{\circ}$ and $40^{\circ}$ but not $60^{\circ}$, and that the magnitude of this effect was related to ecstasy use and was unaffected by cannabis use. In an as yet unpublished study, White, Tran, Brown and Edwards (in preparation) further supported the original theory by showing that the tuning bandwidths of orientation sensitive V1 neurons in ecstasy users were broader than that of non-drug using controls (as determined by a orientation detection masking study). There were also significant differences between these groups in the operation of longer projections within V1 (as determined by a contour

*Address correspondence to this author at the School of Psychology, Australian National University, Australia; Tel: +61 36226 2240; Fax: +61 036226 2883; E-mail: Raimondo.Bruno@utas.edu.au integration task) but only in a subset of ecstasy users who had used cannabis on less than 100 occasions.

Given the seemingly robust effects of ecstasy use on lateral inhibition in V1, investigations were then conducted on higher order functioning within the occipital lobe. Murray and Bruno (in preparation) found no effect of ecstasy use on the operation of V2 neurons - as revealed by Tilt Aftereffect using subjective (illusory) contour stimuli - while again replicating the original effect in V1 neurons using the Tilt Aftereffect with matched real contours. Similarly, White, Tran, Brown and Edwards (in preparation) found no effect of ecstasy use on V5/MT function, using a global motion detection threshold procedure. Given that motion processing deficits have been identified in ecstasy users [3], current studies examining apparent global motion while methodologically controlling for cannabis and methamphetamine use are underway.

\section{CONCLUSIONS}

Overall, these studies support the theory that ecstasyrelated changes to the serotonin system result in the broadening of the tuning bandwidths of orientation sensitive neurons in V1, as well as the functioning of long-range connections within the visual cortex. Curiously, however, despite the hierarchical and sequential processing that occurs in the visual cortex, this effect has not been apparent on tasks reflecting V2 or V5/MT functioning using the measurement techniques investigated to date.

\section{REFERENCES}

[1] Brown J, Edwards M, McKone E, Ward J. A long-term ecstasyrelated change in visual perception. Psychopharmacology (Berl) 2007; 193(3): 437-46.

[2] Dickson C, Bruno R, Brown J. Investigating the role of serotonin in visual orientation processing using an 'ecstasy' (MDMA)-based research model. Neuropsychobiology 2009; 60(3-4): 204-12.

[3] Rizzo M, Lamers CTJ, Sauer CG, Ramaekers JG, Bechara A, Andersen GJ. Impaired perception of self-motion (heading) in abstinent ecstasy and marijuana users. Psychopharmacology 2005; 179: $559-66$

This is an open access article licensed under the terms of the Creative Commons Attribution Non-Commercial License (http://creativecommons.org/licenses/ by-nc/3.0/) which permits unrestricted, non-commercial use, distribution and reproduction in any medium, provided the work is properly cited. 